\title{
Bending Analysis of Laminated \\ Two-Dimensional Piezoelectric Quasicrystal Plates with Functionally Graded Material Properties
}

\author{
YANG LI ${ }^{a, b}$, LIANZHI YANG ${ }^{c}$ AND YANG GAO ${ }^{d, *}$ \\ ${ }^{a}$ College of Engineering, China Agricultural University, Beijing 100083, China \\ ${ }^{b}$ Department of Mechanical and Power Engineering, Yingkou Institute of Technology, Yingkou 115014, China \\ ${ }^{c}$ School of Civil and Resource Engineering, University of Science and Technology Beijing, Beijing 100083, China \\ ${ }^{d}$ College of Science, China Agricultural University, Beijing 100083, China
}

(Received July 2, 2018; in final form January 18, 2019)

\begin{abstract}
This paper presents an exact solution for a functionally graded multilayered two-dimensional piezoelectric quasicrystal plate with simply supported boundary conditions. The material properties of the functionally graded plate are assumed to be exponentially distributed along the thickness direction. Based on the pseudo-Stroh formalism, an exact solution for a functionally graded plate is derived, and the exact solution for the corresponding multilayered case is obtained in terms of the propagator matrix method. Numerical examples show the influences of functionally graded exponential factor, stacking sequence, and phonon-phason coupling coefficient on the plate. The obtained results should be useful for the analysis and design of the functionally graded two-dimensional piezoelectric quasicrystal plate.
\end{abstract}

DOI: 10.12693/APhysPolA.135.426

PACS/topics: quasicrystals, functionally graded materials, piezoelectricity, plates, exact solution

\section{Introduction}

Functionally graded (FG) materials have been widely used in smart structure. Due to the smooth and continuous change of material properties along one direction, they can eliminate stress concentration and improve the bonding strength at the interfaces of the layers. Such smart structure in form of plate made of FG materials and FG piezoelectric materials is very common in engineering. Three-dimensional analysis of thick FG piezoelectric plate was done by utilizing the element-freeGalerkin method [1]. Guo et al. [2] studied the sizedependent behavior of the FG plate under surface load by using the modified couple-stress theory. Sladek et al. [3] proposed a meshless method based on the local PetrovGalerkin approach to investigate the bending behavior of FG plate. Li et al. [4] derived the analytical solution for the axisymmetric problem of FG circular plates. Wu et al. [5] presented the exact solution of FG magnetoelectro-elastic rectangular plates using a modified Pagano method. The elasticity solution of the FG plate subjected to a special family of biharmonic polynomial loading was obtained in terms of three-dimensional theory of elasticity [6]. Liu and Zhong [7], and Zhong and Shang [8] investigated the thermoelastic and piezothermoelectric problems of a FG rectangular plate, respectively. Pan and Han [9] obtained the exact solution for the FGM magneto-electro-elastic plates based on the pseudo-Stroh formalism.

*corresponding author; e-mail: gaoyangg@gmail.com
As new-type solid materials, quasicrystals (QCs) were discovered from the diffraction image of rapidly cooled Al-Mn alloys by Shechtman in early 1980s [10]. QCs have a long-range quasiperiodic translational order and a longrange orientational order [11, 12]. Two lower frequency excitations, which are phonon and phason, are considered in the elastic energy theory of QCs [13]. Attributing to the quasi-periodic structure, QCs possess many desirable properties, such as high hardness, high wear resistance, low adhesion, and low level of porosity [14, 15]. Piezoelectricity has been verified to be a vital physical property of QCs $[16,17]$. Owing to the quasi-periodic atomic arrangement of QCs, they are expected to be used as sensor in intelligent structures, thin films, coating surface of engines, thermal barrier coating, and coating surface of spacecraft's wings [18-20]. Due to the unique properties and potential applications, QCs have attracted much attention in a wide range of research fields, such as defect problems $[19,20]$ and mechanical behavior analysis in the layered smart structure [21-24].

FG piezoelectric $\mathrm{QC}$ (PQC) materials consider FG effect and piezoelectric effect together, which are expected to be used as sensors and actuators to monitor and control the response of structures. Due to the advantages of FG piezoelectric materials and QCs, FG PQCs can not only realize the conversion of mechanical energy and electrical energy, but also enhance the life span and reliability of piezoelectric devices. With the development of preparation technology of FG QCs [25], the study on the smart structure made of FG PQCs becomes particularly important. Motivated by this, an exact solution for a FG layered two-dimensional (2D) PQC plate is presented in this paper. Based on the pseudo-Stroh formalism [26], 
the solution for a FG 2D PQC plate is obtained, and the exact solution for the corresponding multilayered case is derived by using the propagator matrix method. In the numerical examples, the influences of FG exponential factor, stacking sequence, and phonon-phason coupling coefficient on the FG plate subjected to an electric potential load on its top surface are studied.

\section{Fundamental formulations}

The 2D QC refers to a three-dimensional structure with atomic arrangement being quasi-periodic in a plane and periodic along one direction normal to the plane [27]. A 2D PQC with $x_{1}-x_{2}$ plane being the quasi-periodic plane, $x_{3}$ being the periodic and poling directions referring to the Cartesian coordinates $\left(x_{1}, x_{2}, x_{3}\right)$ is considered in this paper. The static equilibrium equations without body forces and electric charges are given by $[28,29]$ :

$$
\sigma_{i j, j}=0, \quad H_{m j, j}=0, \quad D_{j, j}=0,
$$

where $i, j=1,2,3$, and $m=1,2$. $\sigma_{i j}$ and $H_{m j}$ denote the phonon and phason stresses, respectively; $D_{j}$ represents the electric displacements; a subscript comma represents a partial differentiation.

According to the linear elastic theory of QCs [30], the extended strain-displacement relations are

$$
\varepsilon_{i j}=\left(u_{i, j}+u_{j, i}\right) / 2, \quad w_{m j}=w_{m, j}, \quad E_{j}=-\phi_{, j},(2.2)
$$
where $\varepsilon_{i j}$ and $w_{m j}$ represent the phonon and phason strains, respectively; $u_{i}$ and $w_{m}$ are displacements in the phonon and phason fields, respectively; $E_{j}$ and $\phi$ denote the electric fields and electric potential, respectively.

The linear constitutive equations for 2D decagonal PQC with point group $\overline{10} \mathrm{~m} 2$ in Laue 14 can be expressed by the following form [29]:

$$
\begin{aligned}
& \sigma_{11}= \\
& C_{11} \varepsilon_{11}+C_{12} \varepsilon_{22}+C_{13} \varepsilon_{33}+R_{1}\left(w_{11}+w_{22}\right)-e_{31} E_{3}, \\
& \sigma_{22}= \\
& C_{12} \varepsilon_{11}+C_{11} \varepsilon_{22}+C_{13} \varepsilon_{33}-R_{1}\left(w_{11}+w_{22}\right)-e_{31} E_{3}, \\
& \sigma_{33}=C_{13} \varepsilon_{11}+C_{13} \varepsilon_{22}+C_{33} \varepsilon_{33}-e_{33} E_{3}, \\
& \sigma_{23}=\sigma_{32}=2 C_{44} \varepsilon_{23}-e_{15} E_{2}, \\
& \sigma_{31}=\sigma_{13}=2 C_{44} \varepsilon_{13}-e_{15} E_{1}, \\
& \sigma_{12}=\sigma_{21}=2 C_{66} \varepsilon_{12}-R_{1} w_{12}+R_{1} w_{21}, \\
& H_{11}=R_{1}\left(\varepsilon_{11}-\varepsilon_{22}\right)+K_{1} w_{11}+K_{2} w_{22}-d_{112} E_{2}, \\
& H_{22}=R_{1}\left(\varepsilon_{11}-\varepsilon_{22}\right)+K_{1} w_{22}+K_{2} w_{11}+d_{112} E_{2}, \\
& H_{23}=K_{4} w_{23}, \\
& H_{12}=-2 R_{1} \varepsilon_{12}+K_{1} w_{12}-K_{2} w_{21}-d_{112} E_{1}, \\
& H_{13}=K_{4} w_{13},
\end{aligned}
$$

$$
\begin{aligned}
& H_{21}=2 R_{1} \varepsilon_{12}-K_{2} w_{12}+K_{1} w_{21}-d_{112} E_{1}, \\
& D_{1}=2 e_{15} \varepsilon_{13}+d_{112}\left(w_{12}+w_{21}\right)+\kappa_{11} E_{1}, \\
& D_{2}=2 e_{15} \varepsilon_{23}+d_{112}\left(w_{11}-w_{22}\right)+\kappa_{22} E_{2}, \\
& D_{3}=e_{31}\left(\varepsilon_{11}+\varepsilon_{22}\right)+e_{33} \varepsilon_{33}+\kappa_{33} E_{3},
\end{aligned}
$$

where $C_{i j}$ denotes the phonon elastic constants, and $2 C_{66}=C_{11}-C_{12} ; K_{1}, K_{2}$, and $K_{4}$ represent the phason elastic constants; $R_{1}$ is the phonon-phason coupling elastic constant; $e_{15}, e_{31}$, and $e_{33}$ are the piezoelectric constants in the phonon field; $\kappa_{11}, \kappa_{22}$, and $\kappa_{33}$ are the permittivity constants; $d_{112}$ represents the piezoelectric constant in the phason field. Notice that the electroelastic coupling is not considered in the phason field (i.e., $d_{112}=0$ ).

The material properties exponentially varying along the $x_{3}$-direction of the FG $2 \mathrm{D}$ PQC plate have the following distributions:

$$
F\left(x_{3}\right)=F^{0} \mathrm{e}^{\eta x_{3}},
$$

where $F^{0}$ denotes the initial values of material coefficients in Eq. (2.3). $\eta$ is the FG exponential factor indicating the degree of material gradient along the thickness direction.

\section{Problem description and general solution}

Consider a FG layered 2D PQC plate with the Cartesian coordinate system $(x, y, z)$, which is shown in Fig. 1. The local material coordinate system and the global Cartesian coordinate system have the relation $\left(x_{1}, x_{2}, x_{3}\right)=(x, y, z)$. The multilayered plate is simply supported with the geometric sizes of the plate being $x \times y \times z=L_{x} \times L_{y} \times H$. The layer $j$ is bonded by the lower interface $z_{j}$ and the upper interface $z_{j+1}$. The displacements and $z$-direction tractions are assumed to be continuous along the interfaces of the layers. The material properties vary exponentially along the $z$-direction.

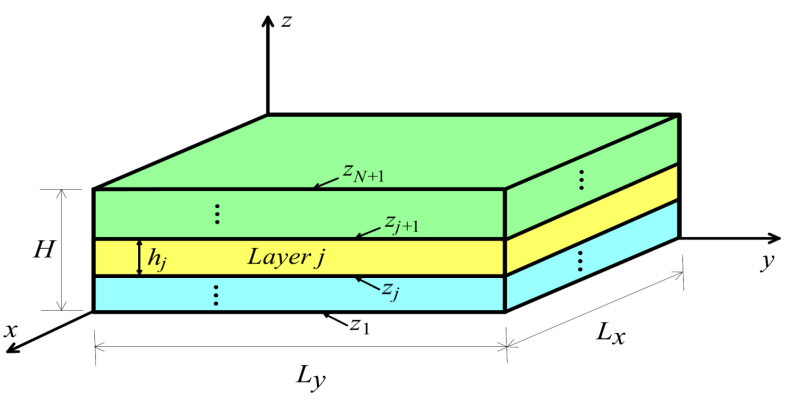

Fig. 1. Schematic configuration of a FG layered 2D PQC plate.

The solution of the extended displacement vector of a FG 2D PQC plate takes the following form: 


$$
\boldsymbol{u}=\left[\begin{array}{c}
u_{x} \\
u_{y} \\
u_{z} \\
w_{x} \\
w_{y} \\
\phi
\end{array}\right]=\mathrm{e}^{s z}\left[\begin{array}{c}
a_{1} \cos p x \sin q y \\
a_{2} \sin p x \cos q y \\
a_{3} \sin p x \sin q y \\
a_{4} \cos p x \sin q y \\
a_{5} \sin p x \cos q y \\
a_{6} \sin p x \sin q y
\end{array}\right],
$$

in which $s, a_{1}, a_{2}, a_{3}, a_{4}, a_{5}$, and $a_{6}$ are unknowns to be determined, and

$$
p=n \pi / L_{x}, \quad q=m \pi / L_{y},
$$

with $n$ and $m$ being two positive integers. Equation (3.1) is one term of a double Fourier series expansion when solving the general solution. It is clear that the displacements and electric potential in Eq. (3.1) satisfy the simply supported boundary conditions, which are shown as follows:

$$
\begin{aligned}
& u_{y}=u_{z}=w_{y}=\phi=\sigma_{x x}=0 \text { at } x=0 \text { and } L_{x}, \\
& u_{x}=u_{z}=w_{x}=\phi=\sigma_{y y}=0 \text { at } y=0 \text { and } L_{y} .
\end{aligned}
$$

With the material properties following an exponent-law distribution along the thickness direction in Eq. (2.4), we substitute Eq. (3.1) into Eq. (2.2), and subsequently into Eq. (2.3), the extended traction vector can be derived as

$$
\boldsymbol{t}=\left[\begin{array}{c}
\sigma_{x z} \\
\sigma_{y z} \\
\sigma_{z z} \\
H_{x z} \\
H_{y z} \\
D_{z}
\end{array}\right]=\mathrm{e}^{(s+\eta) z}\left[\begin{array}{c}
b_{1} \cos p x \sin q y \\
b_{2} \sin p x \cos q y \\
b_{3} \sin p x \sin q y \\
b_{4} \cos p x \sin q y \\
b_{5} \sin p x \cos q y \\
b_{6} \sin p x \sin q y
\end{array}\right] .
$$

The two $6 \times 1$ vectors

$$
\begin{aligned}
& \boldsymbol{a}=\left[a_{1}, a_{2}, a_{3}, a_{4}, a_{5}, a_{6}\right]^{\mathrm{T}}, \\
& \boldsymbol{b}=\left[b_{1}, b_{2}, b_{3}, b_{4}, b_{5}, b_{6}\right]^{\mathrm{T}}
\end{aligned}
$$

are introduced to represent the coefficients in Eqs. (3.1) and (3.4), respectively. The superscript " $\mathrm{T}$ " denotes a vector or matrix transpose. In terms of vector $\boldsymbol{a}$, vector $\boldsymbol{b}$ can be expressed as

$$
\boldsymbol{b}=\left(-\boldsymbol{R}^{\mathrm{T}}+s \boldsymbol{T}\right) \boldsymbol{a}=-\frac{1}{s+\eta}(\boldsymbol{Q}+s \boldsymbol{R}) \boldsymbol{a},
$$

where the matrices $\boldsymbol{Q}, \boldsymbol{R}$ and $\boldsymbol{T}$ can be expressed by the initial material coefficients $C_{i j}^{0}, R_{1}^{0}, K_{i}^{0}, e_{i j}^{0}$, and $d_{m i j}^{0}$.

Substituting all extended stress components into Eq. (2.1), yields the following equation:

$$
\left[\boldsymbol{Q}-\eta \boldsymbol{R}^{\mathrm{T}}+s\left(\boldsymbol{R}-\boldsymbol{R}^{\mathrm{T}}+\eta \boldsymbol{T}\right)+s^{2} \boldsymbol{T}\right] \boldsymbol{a}=\mathbf{0} .
$$

With the aid of Eq. (3.6), Eq. (3.7) can be recast into the following standard eigenrelation:

$$
\boldsymbol{N}\left[\begin{array}{l}
\boldsymbol{a} \\
\boldsymbol{b}
\end{array}\right]=s\left[\begin{array}{l}
\boldsymbol{a} \\
\boldsymbol{b}
\end{array}\right],
$$

where

$$
\boldsymbol{N}=\left[\begin{array}{cc}
\boldsymbol{T}^{-1} \boldsymbol{R}^{\mathrm{T}} & \boldsymbol{T}^{-1} \\
-\boldsymbol{Q}-\boldsymbol{R} \boldsymbol{T}^{-1} \boldsymbol{R}^{\mathrm{T}} & -\boldsymbol{R} \boldsymbol{T}^{-1}-\eta \boldsymbol{I}
\end{array}\right] .
$$

The twelve eigenvalues $s_{i}$ and $s_{i+6}(i=1,2, \ldots, 6)$ are obtained by solving the eigenrelation in Eq. (3.8). The first six eigenvalues $s_{i}$ and the next six eigenvalues $s_{i+6}$ have the relation $s_{i+6}=-s_{i}-\eta$. The eigenvectors $\boldsymbol{a}$ and $\boldsymbol{b}$ corresponding to eigenvalues can be also obtained when solving Eq. (3.8).

Then the general solution for the extended displacement vector in Eq. (3.1) and traction vector in Eq. (3.4) is derived as

$$
\left[\begin{array}{l}
\boldsymbol{u} \\
\boldsymbol{t}
\end{array}\right]=\left[\begin{array}{cc}
\boldsymbol{I} & \mathbf{0} \\
\mathbf{0} & \mathrm{e}^{\eta z} \boldsymbol{I}
\end{array}\right]\left[\begin{array}{ll}
\boldsymbol{A}_{1} & \boldsymbol{A}_{2} \\
\boldsymbol{B}_{1} & \boldsymbol{B}_{2}
\end{array}\right]\left\langle\mathrm{e}^{s^{*} z}\right\rangle\left[\begin{array}{c}
\boldsymbol{D}_{1} \\
\boldsymbol{D}_{2}
\end{array}\right],
$$

where

$$
\begin{aligned}
& \boldsymbol{A}_{1}=\left[\boldsymbol{a}_{1}, \boldsymbol{a}_{2}, \boldsymbol{a}_{3}, \boldsymbol{a}_{4}, \boldsymbol{a}_{5}, \boldsymbol{a}_{6}\right], \\
& \boldsymbol{A}_{2}=\left[\boldsymbol{a}_{7}, \boldsymbol{a}_{8}, \boldsymbol{a}_{9}, \boldsymbol{a}_{10}, \boldsymbol{a}_{11}, \boldsymbol{a}_{12}\right], \\
& \boldsymbol{B}_{1}=\left[\boldsymbol{b}_{1}, \boldsymbol{b}_{2}, \boldsymbol{b}_{3}, \boldsymbol{b}_{4}, \boldsymbol{b}_{5}, \boldsymbol{b}_{6}\right], \\
& \boldsymbol{B}_{2}=\left[\boldsymbol{b}_{7}, \boldsymbol{b}_{8}, \boldsymbol{b}_{9}, \boldsymbol{b}_{10}, \boldsymbol{b}_{11}, \boldsymbol{b}_{12}\right], \\
& \left\langle\mathrm{e}^{s^{*} z}\right\rangle=\operatorname{diag}\left[\mathrm{e}^{s_{i} z}, \mathrm{e}^{\left(-s_{i}-\eta\right) z}\right],
\end{aligned}
$$

with $\boldsymbol{D}_{1}$ and $\boldsymbol{D}_{2}$ being two $6 \times 1$ constant column matrices to be determined according to the boundary conditions, and $s^{*}$ includes all twelve eigenvalues solved from Eq. (3.8).

For the lower surface $z_{j}$ of layer $j$, the constant column matrices $\boldsymbol{D}_{1}$ and $\boldsymbol{D}_{2}$ in Eq. (3.10) can be expressed as

$$
\begin{aligned}
& {\left[\begin{array}{l}
\boldsymbol{D}_{1} \\
\boldsymbol{D}_{2}
\end{array}\right]_{j}=} \\
& \left\langle\mathrm{e}^{-s^{*} z_{j}}\right\rangle\left[\begin{array}{ll}
\boldsymbol{A}_{1} & \boldsymbol{A}_{2} \\
\boldsymbol{B}_{1} & \boldsymbol{B}_{2}
\end{array}\right]^{-1}\left[\begin{array}{cc}
\boldsymbol{I} & \mathbf{0} \\
\mathbf{0} & \mathrm{e}^{-\eta z_{j}} \boldsymbol{I}
\end{array}\right]\left[\begin{array}{l}
\boldsymbol{u} \\
\boldsymbol{t}
\end{array}\right]_{z_{j}} .
\end{aligned}
$$

By virtue of the constant column matrices $\boldsymbol{D}_{1}$ and $\boldsymbol{D}_{2}$ in Eq. (3.12), the physical quantities at the upper surface $z_{j+1}$ of layer $j$ with thickness $h_{j}=z_{j+1}-z_{j}$ are derived as

$$
\left[\begin{array}{l}
\boldsymbol{t} \\
\boldsymbol{t}
\end{array}\right]_{z_{j+1}}=\boldsymbol{P}_{j}\left(h_{j}\right)\left[\begin{array}{c}
\boldsymbol{u} \\
\boldsymbol{t}
\end{array}\right]_{z_{j}},
$$

where

$$
\begin{aligned}
& \boldsymbol{P}_{j}\left(h_{j}\right)=\left[\begin{array}{cc}
\boldsymbol{I} & \mathbf{0} \\
\mathbf{0} & \mathrm{e}^{\eta z_{j+1}} \boldsymbol{I}
\end{array}\right]\left[\begin{array}{ll}
\boldsymbol{A}_{1} & \boldsymbol{A}_{2} \\
\boldsymbol{B}_{1} & \boldsymbol{B}_{2}
\end{array}\right] \\
& \times\left\langle\mathrm{e}^{s^{*}\left(z_{j+1}-z_{j}\right)}\right\rangle\left[\begin{array}{ll}
\boldsymbol{A}_{1} & \boldsymbol{A}_{2} \\
\boldsymbol{B}_{1} & \boldsymbol{B}_{2}
\end{array}\right]^{-1}\left[\begin{array}{cc}
\boldsymbol{I} & \mathbf{0} \\
\mathbf{0} & \mathrm{e}^{-\eta z_{j}} \boldsymbol{I}
\end{array}\right],
\end{aligned}
$$

and $\boldsymbol{P}_{j}\left(h_{j}\right)$ is defined as the propagator matrix of layer $j(j=1,2, \ldots, N)$.

In order to obtain the physical quantities at the top surface $z=H$, the propagator matrix in Eq. (3.14) can be utilized repeatedly. Therefore, we have

$$
\begin{aligned}
& {\left[\begin{array}{l}
\boldsymbol{u} \\
\boldsymbol{t}
\end{array}\right]_{z=H}=} \\
& \quad \boldsymbol{P}_{N}\left(h_{N}\right) \boldsymbol{P}_{N-1}\left(h_{N-1}\right) \ldots \boldsymbol{P}_{2}\left(h_{2}\right) \boldsymbol{P}_{1}\left(h_{1}\right)\left[\begin{array}{c}
\boldsymbol{u} \\
\boldsymbol{t}
\end{array}\right]_{z=0} .
\end{aligned}
$$


It is assumed that the FG 2D PQC plate subjected to an electric potential on its top surface, i.e.,

$$
\boldsymbol{u}(H)=\left[0,0,0,0,0, \phi_{0} \sin p x \sin q y\right]^{\mathrm{T}},
$$

and all other traction on the bottom and top surfaces are assumed to be zero. Then, the exact solution in Eq. (3.15) can be recast as

$$
\left[\begin{array}{c}
\boldsymbol{u}(H) \\
\mathbf{0}
\end{array}\right]=\left[\begin{array}{ll}
\boldsymbol{C}_{1} & \boldsymbol{C}_{2} \\
\boldsymbol{C}_{3} & \boldsymbol{C}_{4}
\end{array}\right]\left[\begin{array}{c}
\boldsymbol{u}(0) \\
\mathbf{0}
\end{array}\right],
$$

where $\boldsymbol{C}_{1}, \boldsymbol{C}_{2}, \boldsymbol{C}_{3}$ and $\boldsymbol{C}_{4}$ are the submatrices of the propagator matrix in Eq. (3.15). Solving Eq. (3.17), the unknown extended displacements at the bottom surface of the plate can be obtained as

$$
\boldsymbol{u}(0)=\boldsymbol{C}_{1}^{-1} \boldsymbol{u}(H) .
$$

The unknown quantities at any $z$-level $(0<z \leq H)$ can be obtained in terms of Eqs. (3.15) and (3.18). For the given $z$-level, the top one $z=H$ in Eq. (3.15) should be replaced.

\section{Numerical examples}

In this section, we consider a FG 2D PQC plate subjected to an electric potential load on its top surface to determine the influences of exponential factor $\eta$, stacking sequence, and phonon-phason coupling coefficient on the phonon, phason, and electric fields. Two different stacking sequences are $\mathrm{C} / \mathrm{PQC} / \mathrm{C}$ and $\mathrm{PQC} / \mathrm{C} / \mathrm{PQC}$, respectively. $\mathrm{C}$ represents the piezoelectric crystal material $\mathrm{BaTiO}_{3}$, and $\mathrm{PQC}$ is $\mathrm{Al}-\mathrm{Ni}-\mathrm{Co}$. The material coefficients for $\mathrm{BaTiO}_{3}$ are the following [31]: $C_{11}^{0}=166, C_{12}^{0}=77$, $C_{13}^{0}=78, C_{33}^{0}=162, C_{44}^{0}=43, e_{15}^{0}=11.6, e_{31}^{0}=-4.4$, $e_{33}^{0}=18.6, d_{112}^{0}=0, \kappa_{11}^{0}=\kappa_{22}^{0}=11.2, \kappa_{33}^{0}=12.6$. The units are: $C_{i j}^{0}$ in $10^{9} \mathrm{~N} / \mathrm{m}^{2}, e_{i j}^{0}, d_{m i j}^{0}$ in $\mathrm{C} / \mathrm{m}^{2}$ and $\kappa_{i j}^{0}$ in $10^{-9} \mathrm{C}^{2} /\left(\mathrm{N} \mathrm{m}^{2}\right)$. Because it is difficult to mea- sure the piezoelectric and permittivity constants of QCs, 0.5 time piezoelectric constant and 2 time permittivity constant of $\mathrm{BaTiO}_{3}$ are taken for $\mathrm{Al}-\mathrm{Ni}-\mathrm{Co}$, which is shown as [32]: $C_{11}^{0}=234.33, C_{12}^{0}=57.41, C_{13}^{0}=66.63$, $C_{33}^{0}=232.22, C_{44}^{0}=70.19, R_{1}^{0}=8.846, K_{1}^{0}=122$, $K_{2}^{0}=24, K_{4}^{0}=12, e_{15}^{0}=5.8, e_{31}^{0}=-2.2, e_{33}^{0}=9.3$, $d_{112}^{0}=0, \kappa_{11}^{0}=\kappa_{22}^{0}=22.4, \kappa_{33}^{0}=25.2$. The corresponding units are: $C_{i j}^{0}, R_{1}^{0}, K_{i}^{0}$ in $10^{9} \mathrm{~N} / \mathrm{m}^{2}, e_{i j}^{0}, d_{m i j}^{0}$ in $\mathrm{C} / \mathrm{m}^{2}$ and $\kappa_{i j}^{0}$ in $10^{-9} \mathrm{C}^{2} /\left(\mathrm{N} \mathrm{m}^{2}\right)$. According to Refs. [32, 33], the following boundary condition in phason field should be satisfied at the interface between $\mathrm{PQC}$ and piezoelectric crystal: $H_{m j}=0$. In order to deal with the layered case and avoid the singular matrices, $10^{-8}$ of the corresponding $K_{i}$ value in PQCs and $R_{1}=0$ are taken for piezoelectric crystals. In this way, the interface boundary condition can be very closely satisfied. As for the piezoelectric crystal layer, notice that phason stresses are very close to zero (relative to those in PQC layer); phason displacements indicating the local rearrangement of the atoms have no physical meaning at all, so they should be zero.

QC layer of millimeter-scale has been fabricated and utilized in industry [34], so the dimensions of the FG plate are selected to be $L_{x} \times L_{y} \times H=10 \mathrm{~mm} \times 10 \mathrm{~mm}$ $\times 3 \mathrm{~mm}$, which can be regarded as macro-size [35]. As for the multilayered plate, it has an equal thickness of $1 \mathrm{~mm}$ for each layer. $n=m=1$, and the amplitude $\phi_{0}=1 \mathrm{~V}$. The physical quantity responses along the thickness direction of the plates are shown at the fixed horizontal coordinate $(x, y)=\left(0.75 L_{x}, 0.75 L_{y}\right)$. Different exponential factors $\eta$, which are $-2,0$, and 2 , are chosen for comparison. The results with no functionally graded effect and piezoelectric effect have been found to be in good agreement with the results in Ref. [32].
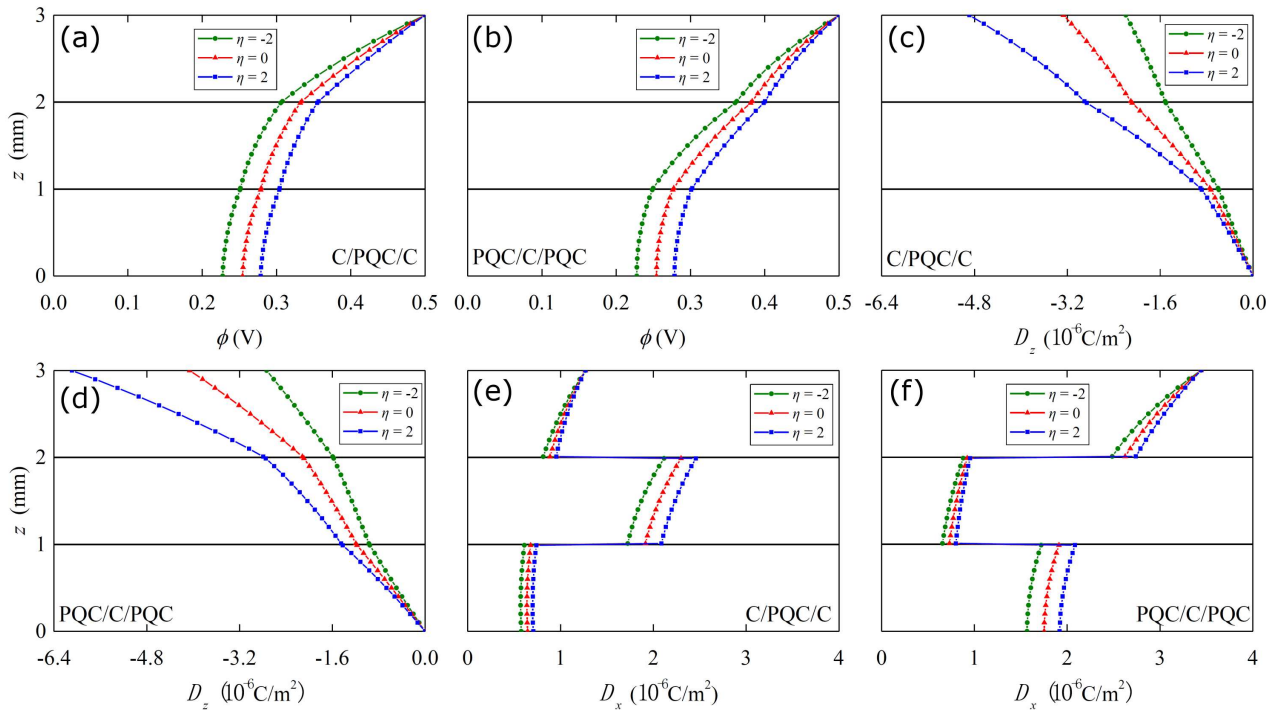

Fig. 2. Variation of the electric potential and electric displacements along the $z$-direction of a FG sandwich plate with different exponential factors. (a) $\phi$ in $\mathrm{C} / \mathrm{PQC} / \mathrm{C}$ plate, (b) $\phi$ in $\mathrm{PQC} / \mathrm{C} / \mathrm{PQC}$ plate, (c) $D_{z}$ in $\mathrm{C} / \mathrm{PQC} / \mathrm{C}$ plate, $(\mathrm{d}) D_{z}$ in $\mathrm{PQC} / \mathrm{C} / \mathrm{PQC}$ plate, (e) $D_{x}$ in $\mathrm{C} / \mathrm{PQC} / \mathrm{C}$ plate, (f) $D_{x}$ in $\mathrm{PQC} / \mathrm{C} / \mathrm{PQC}$ plate. 

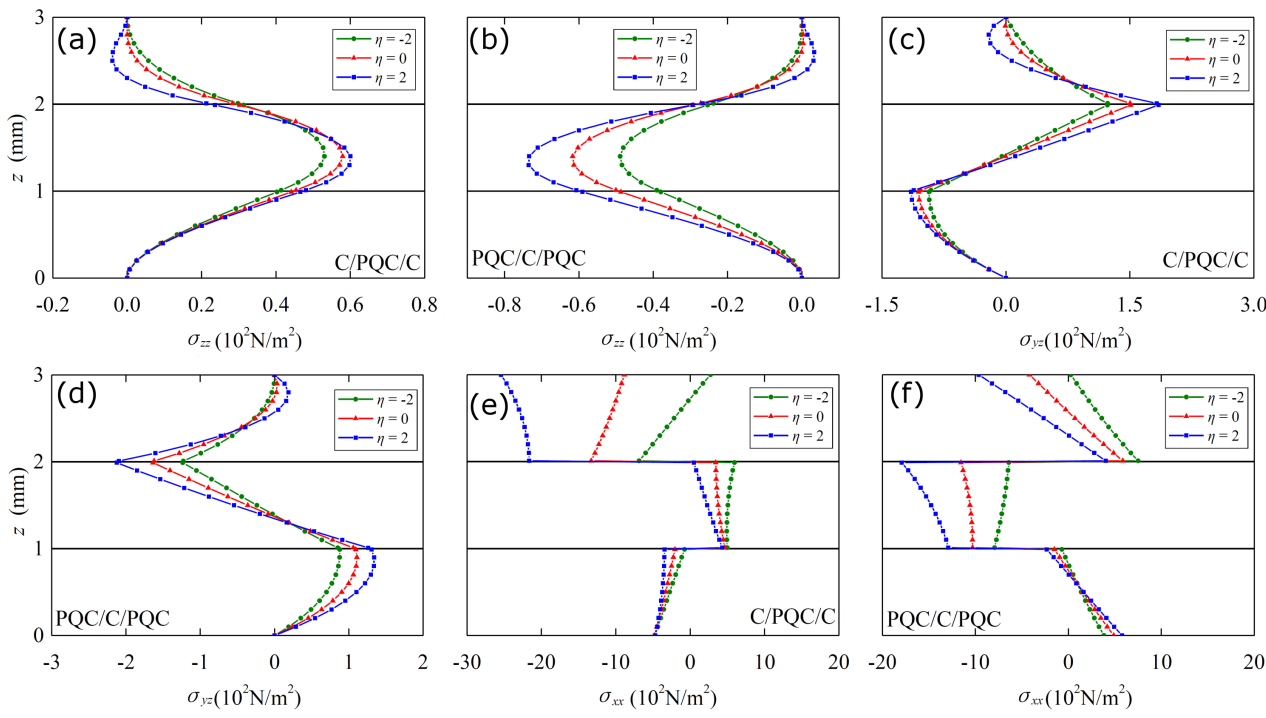

Fig. 3. Variation of the phonon stresses along the $z$-direction of a FG sandwich plate with different exponential factors. (a) $\sigma_{z z}$ in $\mathrm{C} / \mathrm{PQC} / \mathrm{C}$ plate, (b) $\sigma_{z z}$ in $\mathrm{PQC} / \mathrm{C} / \mathrm{PQC}$ plate, (c) $\sigma_{y z}$ in $\mathrm{C} / \mathrm{PQC} / \mathrm{C}$ plate, (d) $\sigma_{y z}$ in $\mathrm{PQC} / \mathrm{C} / \mathrm{PQC}$ plate, (e) $\sigma_{x x}$ in $\mathrm{C} / \mathrm{PQC} / \mathrm{C}$ plate, (f) $\sigma_{x x}$ in $\mathrm{PQC} / \mathrm{C} / \mathrm{PQC}$ plate.
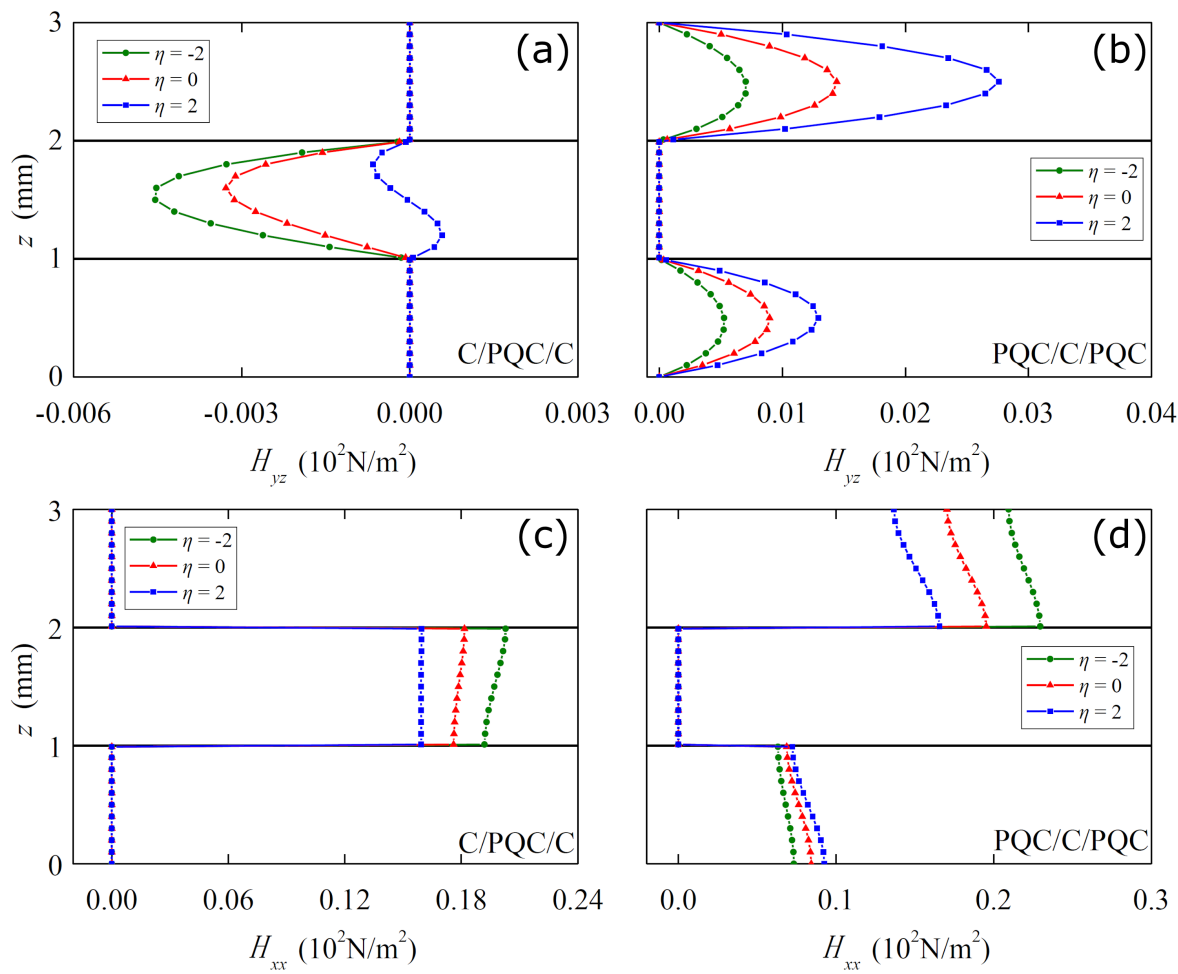

Fig. 4. Variation of the phason stresses along the $z$-direction of a FG sandwich plate with different exponential factors. (a) $H_{y z}$ in $\mathrm{C} / \mathrm{PQC} / \mathrm{C}$ plate, (b) $H_{y z}$ in $\mathrm{PQC} / \mathrm{C} / \mathrm{PQC}$ plate, (c) $H_{x x}$ in $\mathrm{C} / \mathrm{PQC} / \mathrm{C}$ plate, (d) $H_{x x}$ in $\mathrm{PQC} / \mathrm{C} / \mathrm{PQC}$ plate.

The satisfaction of the applied boundary conditions at the top and bottom surfaces can be found from Fig. 2a,b for electric potential $\phi$, Fig. 3a-d for phonon stresses $\sigma_{z z}$ and $\sigma_{y z}$, Fig. $4 \mathrm{a}$,b for phason stress $H_{y z}$, which can partially prove the correctness of the exact solution.

Figure 2 presents the influence of exponential factor $\eta$ on the electric potential and electric displacements for two sandwich plates. Due to the same loading, the values of electric potential $\phi$ in Fig. 2a,b are equal at the top surface of the plates, while the magnitudes of $\phi$ in other $z$-levels of the plates increase with increase of exponential factor $\eta$. For a given exponential factor $\eta$, the magnitude of $\phi$ in $\mathrm{PQC} / \mathrm{C} / \mathrm{PQC}$ plate is a little larger than that in $\mathrm{C} / \mathrm{PQC} / \mathrm{C}$ plate. The electric displacements $D_{z}$ and $D_{x}$ are shown in Fig. 2c-f, and the orders of their magnitudes are the same. Furthermore, $D_{z}$ decreases with 
decrease of exponential factor $\eta$, and the similar trend can be observed from $D_{x} . D_{z}$ and $D_{x}$ are easily affected by stacking sequence.

The variation of phonon stresses along the $z$-direction with different exponential factors $\eta$ and stacking sequences are shown in Fig. 3. For $\sigma_{z z}$ and $\sigma_{y z}$ in Fig. 3a-d, they change with exponential factor $\eta$. It can be found that the maximum value of $\sigma_{z z}$ occurs at the middle layer of both sandwich plates, while the maximum value of $\sigma_{y z}$ is observed at the interfaces of two sandwich plates. The values of the in-plane stresses $\sigma_{x x}$ in Fig. 3e-f are much larger than $\sigma_{z z}$ and $\sigma_{y z}$. It is observed that the influence of exponential factor on $\sigma_{x x}$ in Fig. 3e-f becomes much more obvious with increase of the thickness of plates.
Figure 4 shows the variations of the phason stresses of the two sandwich plates with different exponential factors $\eta$. It is obvious that phason stresses $H_{y z}$ and $H_{x x}$ in Fig. $4 \mathrm{a}-\mathrm{d}$ are zero in the crystal layers, because no phason field exists in crystal. The significant influence of exponential factor $\eta$ on $H_{y z}$ can be observed from Fig. 4a,b. The different influences of stacking sequence on the plates can be observed from $H_{x x}$ in Fig. 4c, d. Furthermore, $H_{x x}$ in $\mathrm{C} / \mathrm{PQC} / \mathrm{C}$ plate decreases with increase of exponential factor $\eta$. The similar trend of $H_{x x}$ in $\mathrm{C} / \mathrm{PQC} / \mathrm{C}$ plate can be found from the top layer in $\mathrm{PQC} / \mathrm{C} / \mathrm{PQC}$ plate, while the bottom layer in $\mathrm{PQC} / \mathrm{C} / \mathrm{PQC}$ plate follows the opposite trend.
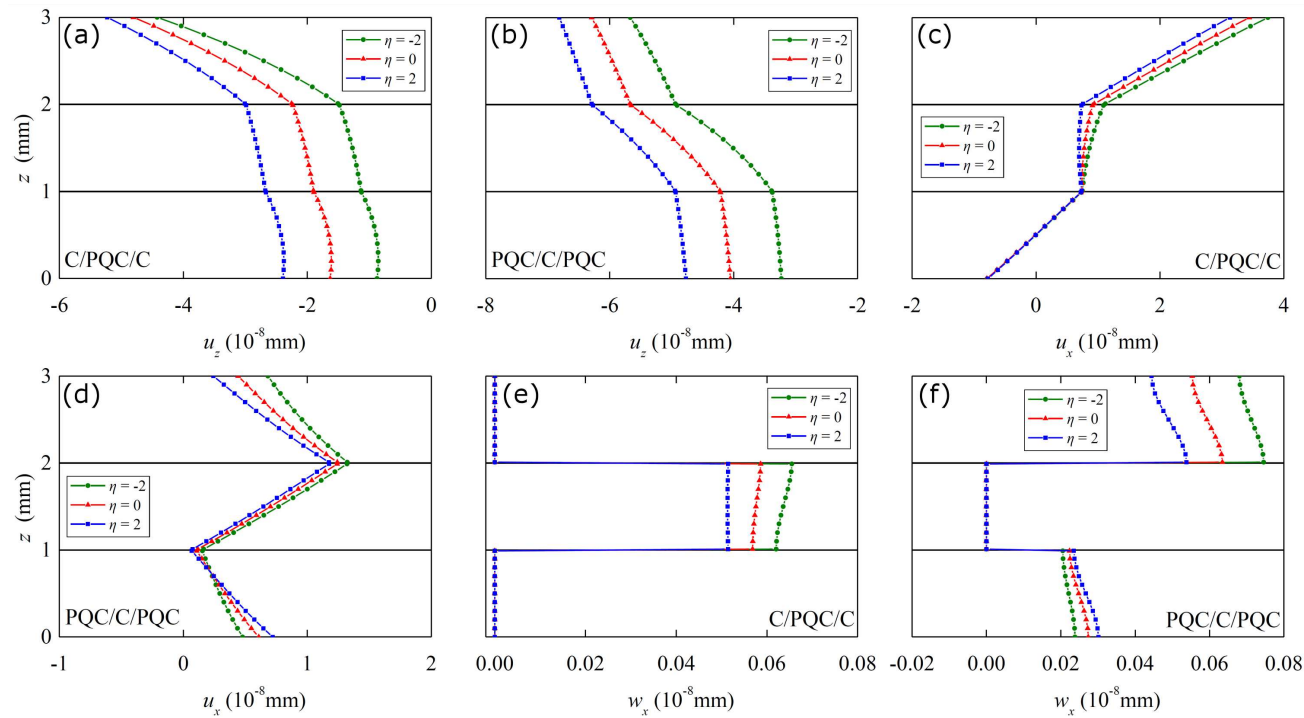

Fig. 5. Variation of the phonon and phason displacements along the $z$-direction of a FG sandwich plate with different exponential factors. (a) $u_{z}$ in $\mathrm{C} / \mathrm{PQC} / \mathrm{C}$ plate, (b) $u_{z}$ in $\mathrm{PQC} / \mathrm{C} / \mathrm{PQC}$ plate, (c) $u_{x}$ in $\mathrm{C} / \mathrm{PQC} / \mathrm{C}$ plate, (d) $u_{x}$ in $\mathrm{PQC} / \mathrm{C} / \mathrm{PQC}$ plate, (e) $w_{x}$ in $\mathrm{C} / \mathrm{PQC} / \mathrm{C}$ plate, (f) $w_{x}$ in $\mathrm{PQC} / \mathrm{C} / \mathrm{PQC}$ plate.
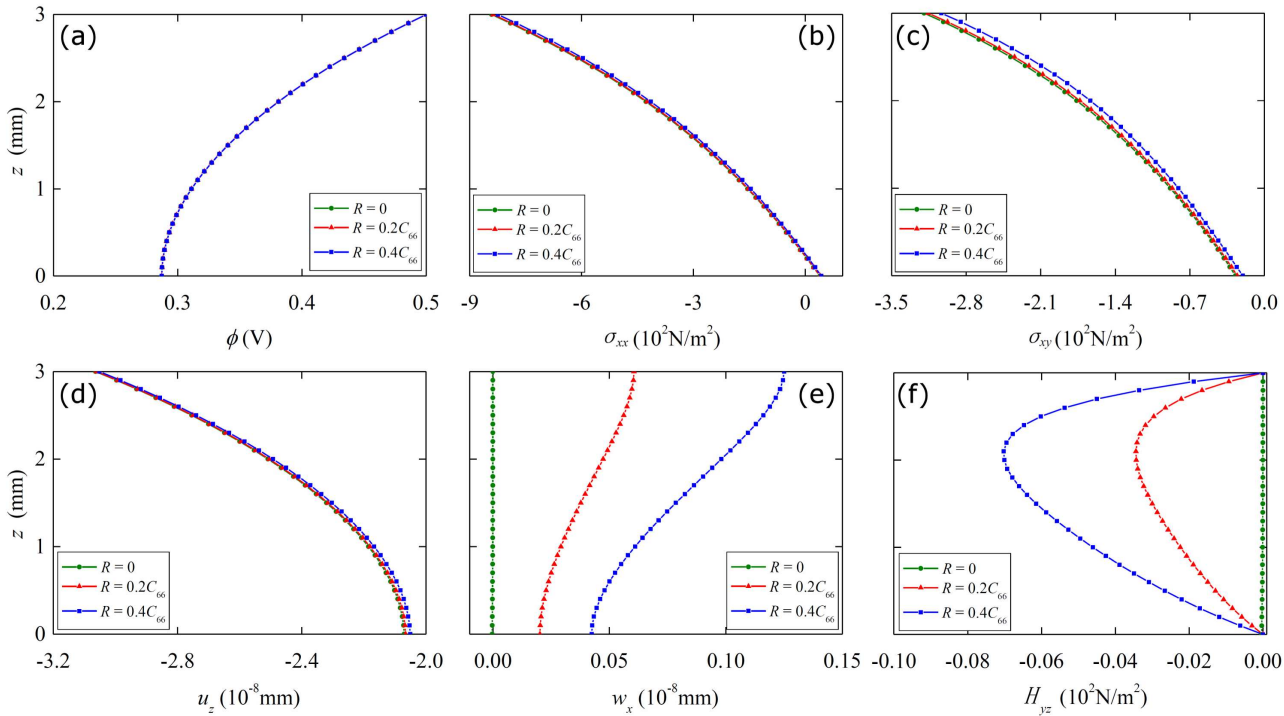

Fig. 6. Variation of the physical quantities along the $z$-direction of a single layer FG plate with different phonon-phason coupling coefficients. (a) $\phi$, (b) $\sigma_{x x}$, (c) $\sigma_{x y}$, (d) $u_{z}$, (e) $w_{x}$, (f) $H_{y z}$. 
Figure 5 shows the distribution of the phonon and phason displacements along the thickness direction for different exponential factors $\eta$ and stacking sequences. Phonon displacement $u_{z}$ in Fig. 5a, b increases with increasing exponential factor $\eta$. For a given exponential factor $\eta$, the stacking sequence also has a great effect on $u_{z}$. Similar to the behavior of $u_{z}$, the influence of exponential factor $\eta$ on phonon displacement $u_{x}$ in Fig. $5 \mathrm{c}, \mathrm{d}$ is obvious. Due to no phason field in crystal, phason displacement $w_{x}$ in crystal layers (Fig. 5e, f) is zero. The dependence of $w_{x}$ on exponential factor $\eta$ is great, and the different trends of $w_{x}$ with related to $\eta$ are found from different layers of the plates. In other words, $w_{x}$ increases with decreasing exponential factor $\eta$ at the middle layer in $\mathrm{C} / \mathrm{PQC} / \mathrm{C}$ plate and the top layer in $\mathrm{PQC} / \mathrm{C} / \mathrm{PQC}$ plate, while the opposite trend is found for $w_{x}$ at the bottom layer in $\mathrm{PQC} / \mathrm{C} / \mathrm{PQC}$ plate.

In order to show the influence of phonon-phason coupling coefficient on the single layer $\mathrm{FG}$ QC plate, 0, $0.2 C_{66}, 0.4 C_{66}$ are taken as phonon-phason coupling coefficient, respectively. The exponential factor is $\eta=2$.

Figure 6 shows the variations of the physical quantities with different phonon-phason coupling coefficients. Phonon-phason coupling coefficient has little effect on electric potential $\phi$ (Fig. 6a). The in-plane phonon stresses $\sigma_{x x}$ (Fig. 6b), $\sigma_{x y}$ (Fig. 6c) and phonon displacement $u_{z}$ (Fig. 6d) decrease slightly with increase of phonon-phason coupling coefficient, which may be attributed to the good wear resistance of QCs. Phason displacement $w_{x}$ (Fig. 6e) and phason stress $H_{y z}$ (Fig. 6f) are more sensitive to the phonon-phason coupling coefficient in comparison to displacement and stress in the phonon field.

\section{Conclusions}

The static deformation of a FG layered 2D PQC simply supported plate, subjected to an electric potential load on its top surface, is investigated. The exact solution for FG layered 2D PQC plates is obtained in terms of the pseudo-Stroh formalism in conjunction with the propagator matrix method. The influences of exponential factor, stacking sequence and phonon-phason coupling coefficient on the FG plate are studied in numerical examples. Numerical results show that all the physical quantities in the phonon, phason, and electric fields are sensitive to the exponential factor and stacking sequence. An increase of phonon-phason coupling coefficient leads to an increase of phason displacements and stresses, and a slight decrease of in-plane stresses in the phonon field. The features obtained show that the introduction of PQCs in smart structures may enhance the carrying capacity and the wear resistance of laminated plates in comparison to traditional piezoelectric materials. The numerical results should be useful for the analysis, design, and numerical simulation of FG PQC plates.

\section{Acknowledgments}

The work is supported by the National Natural Science Foundation of China (Nos. 51704015 and 11472299), China Agricultural University Education Foundation (No. 1101-240001), and China Scholarship Council.

\section{References}

[1] S. Mikaeeli, B. Behjat, Compos. Struct. 154, 591 (2016).

[2] J.H. Guo, J.Y. Chen, E. Pan, Int. J. Eng. Sci. 106, 110 (2016).

[3] J. Sladek, V. Sladek, S. Krahulec, C.S. Chen, D.L. Young, Mech. Adv. Mater. Struct. 22, 479 (2015).

[4] X.Y. Li, H.J. Ding, W.Q. Chen, P.D. Li, J. Appl. Mech. 80, 041007 (2013).

[5] C.P. Wu, S.J. Chen, K.H. Chiu, Mech. Res. Commun. 37, 54 (2010).

[6] B. Yang, H.J. Ding, W.Q. Chen, Appl. Math. Model. 36, 488 (2012).

[7] W.X. Liu, Z. Zhong, Acta Mech. Solid. Sin. 24, 241 (2011).

[8] Z. Zhong, E.T. Shang, J. Int. Mater. Syst. Str. 16 643 (2005).

[9] E. Pan, F. Han, Int. J. Eng. Sci. 43, 321 (2005).

[10] D. Shechtman, I. Blech, D. Gratias, J.W. Cahn, Phys. Rev. Lett. 53, 1951 (1984).

[11] D. Levine, P.J. Steinhardt, Phys. Rev. Lett. 53, 2477 (1984).

[12] J.H. Guo, J. Yu, Y.M. Xing, Mech. Res. Commun. 52, 40 (2013).

[13] L.Z. Yang, F.M. He, Y. Gao, Acta Phys. Pol. A 126. 471 (2014).

[14] D.V. Louzguine-Luzgin, A. Inoue, Ann. Rev. Mater. Res. 38, 403 (2008).

[15] M.H. Zhao, H.Y. Dang, C.Y. Fan, Z.T. Chen, Eng. Fract. Mech. 179, 59 (2017).

[16] C.Z. Hu, R.H. Wang, D.H. Ding, W.G. Yang, Phys. Rev. B 56, 2463 (1997).

[17] G. Altay, M.C. Dökmeci, Int. J. Solids Struct. 49, 3255 (2012)

[18] T.Y. Fan, Mathematical Theory of Elasticity of Quasicrystals and Its Applications, Springer, Heidelberg 2011.

[19] Y. Gao, A. Ricoeur, L.L. Zhang, L.Z. Yang, Arch. Appl. Mech. 84, 1103 (2014).

[20] P.D. Li, X.Y. Li, G.Z. Kang, Mech. Res. Commun. 70, 72 (2015).

[21] Y. Li, L.Z. Yang, Y. Gao, E. Pan, J. Intel. Mater. Syst. Str. 29, 2660 (2018).

[22] Y. Li, L.Z. Yang, Y. Gao, Z. Angew. Math. Mech. 98, 1585 (2018).

[23] L.Z. Yang, Y. Li, Y. Gao, E. Pan, Appl. Math. Model. 63, 203 (2018)

[24] J. Sladek, K. Sladek, C. Zhang, M. Wünsche, Eur. J. Mech. A Solid. 49, 518 (2015). 
[25] K.C. Chan, N.S. Qu, D. Zhu, T.I. Met. Finish. 80, 210 (2002).

[26] E. Pan, J. Appl. Mech. 68, 608 (2001).

[27] Y. Gao, A. Ricoeur, Proc. R. Soc. A 467, 2622 (2011).

[28] C.Z. Hu, R.H. Wang, D.H. Ding, Rep. Prog. Phys. 63, 1 (2000).

[29] L.Z. Yang, Y. Li, Y. Gao, E. Pan, N. Waksmanski, Compos. Struct. 171, 198 (2017).

[30] D.H. Ding, W.G. Yang, C.Z. Hu, R.H. Wang, Phys. Rev. B 48, 7003 (1993).
[31] J.S. Lee, L.Z. Jiang, Int. J. Solids Struct. 33, 977 (1996).

[32] L.Z. Yang, Y. Gao, E. Pan, N. Waksmanski, Int. J. Solids Struct. 51, 1737 (2014).

[33] T.Y. Fan, L.Y. Xie, L. Fan, Q.Z. Wang, Chin. Phys. B 20, 076102 (2011).

[34] J.M. Dubois, Chem. Soc. Rev. 41, 6760 (2012).

[35] Q.H. Qin, Q.S. Yang, Macro-Micro Theory on Multifield Coupling Behavior of Heterogeneous Materials, Springer, Heidelberg 2009 